\title{
INFRARED ARRAY CAMERA (IRAC) IMAGING OF THE LOCKMAN HOLE
}

\author{
J.-S. Huang, ${ }^{1}$ P. Barmby, ${ }^{1}$ G. G. Fazio, ${ }^{1}$ S. P. Willner, ${ }^{1}$ G. Wilson ${ }^{2}$ D. Rigopoulou, ${ }^{3}$ A. Alonso-Herrero, ${ }^{4}$ H. Dole, ${ }^{4,5}$ \\ E. Egami, ${ }^{4}$ E. Le Floc'h, ${ }^{4}$ C. Papovich, ${ }^{4}$ P. G. Pérez-González, ${ }^{4}$ J. Rigby, ${ }^{4}$ C. W. Engelbracht, ${ }^{4}$ K. Gordon, ${ }^{4}$ \\ D. Hines, ${ }^{4}$ M. Rieke, ${ }^{4}$ G. H. Rieke, ${ }^{4}$ K. Meisenheimer, ${ }^{6}$ and S. Miyazaki ${ }^{7}$ \\ Received 2004 March 26; accepted 2004 April 29
}

\begin{abstract}
IRAC imaging of a 4.'7 $\times 4 .^{\prime} 7$ area in the Lockman Hole detected over 400 galaxies in the IRAC 3.6 and $4.5 \mu \mathrm{m}$ bands, 120 in the $5.8 \mu \mathrm{m}$ band, and 80 in the $8.0 \mu \mathrm{m}$ band in 30 minutes of observing time. Color-color diagrams suggest that about half of these galaxies are at redshifts $0.6<z<1.3$ with about a quarter at higher redshifts $(z>1.3)$. We also detect IRAC counterparts for six of the seven SCUBA sources and all nine XMM-Newton sources in this area. The detection of the counterparts of the SCUBA sources and galaxies at $z>1.3$ demonstrates the ability of IRAC to probe the universe at very high redshifts.
\end{abstract}

Subject headings: cosmology: observations — galaxies: evolution — galaxies: high-redshift — infrared: galaxies - surveys

\section{INTRODUCTION}

Much effort has gone into deep optical imaging with hope of detecting galaxies at high redshifts (Koo \& Kron 1992; Ellis 1997). Obtaining redshifts for faint, optically selected galaxies require long observations on $\geq 8 \mathrm{~m}$ class telescopes, but most of the galaxies identified are at intermediate redshifts $(z \sim 1$; Cowie et al. 1996; Cohen et al. 1999; Cimatti et al. 2002). Applying faintness criteria to visible-wavelength (UBVRI) surveys is not an efficient way of selecting highredshift galaxies unless color selection methods such as the Lyman break technique (Steidel et al. 2003) or extreme red color selection (Thompson et al. 1999) are applied. Furthermore, optical studies of high-redshift galaxies are heavily affected by dust extinction because rest-frame UV photons are being observed (Steidel et al. 2003).

By contrast, far-infrared and submillimeter surveys are efficient tools for generating samples of high-redshift starburst galaxies. For example, SCUBA on the James Clerk Maxwell Telescope (JCMT) has made it possible to carry out deep extragalactic surveys in the submillimeter band. Now it is known that most SCUBA sources are ultraluminous infrared galaxies (ULIRGs) at high redshifts. They are detected in the submillimeter band, because the infrared luminosity peak at about $100-200 \mu \mathrm{m}$ shifts to the submillimeter and millimeter band at $z>2$ (Chapman et al. 2003; Ivison et al. 2002; Smail et al. 2002).

Little is known about the properties of mid-infrared selected samples, although in principle they should have many advantages. Mid-infrared observations of high-redshift galaxies

\footnotetext{
${ }^{1}$ Harvard-Smithsonian Center for Astrophysics, 60 Garden Street, Cambridge, MA 02138

2 Spitzer Science Center, California Institute of Technology, 1200 East California, Pasadena, CA 91125.

3 Department of Astrophysics, Oxford University, Keble Road, Oxford, OX1 3RH, UK.

4 Steward Observatory, University of Arizona, Tucson, AZ 85721.

5 Institut d'Astrophysique Spatiale, bat 121, Universite Paris Sud, F-91405 Orsay Cedex, France.

6 Max-Planck-Institut für Astronomie, Königstuhl 17, D-69117 Heidelberg, Germany.

${ }^{7}$ Subaru Telescope, National Astronomical Observatory of Japan, 650 North A'ohoku Place, Hilo, HI 96720.
}

detect light emitted at 1-2 $\mu \mathrm{m}$ near the peak of the stellar emission. At these wavelengths the emission is only modestly affected by dust extinction. The difficulty is that a space observatory is essential for sensitive mid-infrared observations. The Infrared Space Observatory (ISO; Kessler 1996) carried out the first deep infrared extragalactic surveys and detected galaxies at redshifts up to $z=1.5$ in the mid-infrared bands (Oliver et al. 1997; Elbaz et al. 1999; Aussel et al. 1999; Genzel \& Cesarsky 2000; Dole et al. 2001; Sato et al. 2003). With its combination of high sensitivity and high spatial resolution in four mid-infrared bands, the Infrared Array Camera (IRAC; Fazio et al. 2004) on the Spitzer Space Telescope can carry out deeper and larger surveys and should be able to detect galaxies at $z>3$ (Barmby et al. 2004).

This paper reports the results of a Spitzer survey carried out during the Spitzer in-orbit checkout (IOC) period. Observations were taken as one of the Early Release Observations to demonstrate the sensitivity of Spitzer and its capability of detecting galaxies at high redshifts. The program included both IRAC and Multiband Imaging Photometer for Spitzer (MIPS) observations of the same Lockman Hole field. Egami et al. (2004) and Le Floc'h et al. (2004) present the results of the MIPS observations of the same field. The survey area centered on R.A. $(\mathrm{J} 2000)=10^{\mathrm{h}} 51^{\mathrm{m}} 56^{\mathrm{s}}$, decl. $(\mathrm{J} 2000)=57^{\circ} 25^{\prime} 32^{\prime \prime}$ has been covered by surveys at many other wavelengths, including SCUBA, XMM-Newton, and deep optical/NIR imaging. Of seven SCUBA sources in the survey area, IRAC detected six in the 3.6 and $4.5 \mu \mathrm{m}$ bands and four in the 5.8 and $8.0 \mu \mathrm{m}$ bands. IRAC also detected mid-infrared counterparts of all nine $X M M-N e w t o n$ sources. Analysis of the Spitzer counterparts of the SCUBA, XMM-Newton, and MAMBO sources are presented by Egami et al. (2004), Alonso-Herrero et al. (2004), and Ivison et al. (2004).

\section{OBSERVATIONS AND DATA REDUCTION}

The observations ${ }^{8}$ covered one field of view in all four IRAC bands. The observations used the $30 \mathrm{~s}$ frame time and 25 dithers per pointing, giving an exposure time (i.e., effective on-chip charge accumulation time after accounting for readout

\footnotetext{
${ }^{8}$ Spitzer Program ID 1077, executed 2003 November 20.
} 
TABLE 1

Number of Objects Detected in the IRAC Bands

\begin{tabular}{crrrc}
\hline \hline $\begin{array}{c}\text { Band } \\
(\mu \mathrm{m})\end{array}$ & Galaxies & Stars & Total & Magnitude Limit $(5 \sigma, \mathrm{AB})$ \\
\hline $3.6 \ldots \ldots \ldots \ldots$ & 419 & 13 & 432 & 23.73 \\
$4.5 \ldots \ldots \ldots \ldots$. & 403 & 13 & 413 & 23.77 \\
$5.8 \ldots \ldots \ldots \ldots$. & 120 & 5 & 125 & 21.90 \\
$8.0 \ldots \ldots \ldots \ldots$. & 80 & 5 & 85 & 21.68 \\
$24.0 \ldots \ldots \ldots \ldots$ & 32 & 0 & 32 & 18.15 \\
\hline
\end{tabular}

overheads) of $670 \mathrm{~s}$ in each band. We used a shorter frame time and many dithers to be able to reject cosmic rays effectively. Because of the dithering, the area covered to full depth was $4.7 \times 4.7$.

Data processing began with the Basic Calibrated Data (BCD) delivered by the Spitzer Science Center (SSC). These data include flat-field corrections, dark subtraction, and linearity and flux calibrations. Additional steps included pointing refinement, distortion correction, and mosaicking. Cosmic rays were rejected during the last step by sigma-clipping.

Source extraction used DAOPHOT because the images are so crowded in the 3.6 and $4.5 \mu \mathrm{m}$ bands even for the short observations reported here. Given the 1".8 FWHM of the point spread function (Fazio et al. 2004) in both bands, almost all faint sources are pointlike. We used a signal-to-noise ratio of 2.5 , and a PSF with full width at half-maximum (FHWM) of $1 " 8,1.8,2 . " 0$, and 2.0 in the $3.6,4.5,5.8$, and $8.0 \mu \mathrm{m}$ bands respectively as parameters for DAOPHOT.

Star/galaxy separation was based on morphology in deep $R$-band images taken with the Suprime camera on the Subaru Telescope and on a $(V-I$ vs. $I-K)$ color-color diagram (Huang et al. 1997; Wilson et al. 2001). We identified 13 stars in the field with $K \leq 20.5$. Though fainter stars may be missed, their density is extremely low (Glazebrook et al. 1994). Table 1 gives the numbers of galaxies and stars detected in each band along with the limiting flux. The number of galaxies detected in the MIPS $24 \mu \mathrm{m}$ band (Egami et al. 2004; Le Floc'h et al. 2004) is also in Table 1. Fewer sources were detected in the two long-wavelength bands because of the high zodiacal background at those wavelengths. All objects detected at 5.8 or $8.0 \mu \mathrm{m}$ are detected at both 3.6 and $4.5 \mu \mathrm{m}$. There are about 25,000 "beams" in the field, and the $3.6 \mu \mathrm{m}$ source counts thus correspond to 57 beams per source.

\section{THE PROPERTIES OF GALAXIES DETECTED BY IRAC}

With no redshifts available for most of the IRAC galaxy sample, we rely on color-color diagrams to estimate redshifts. In the rest near-infrared wavelength range, the most important spectral feature is the $1.6 \mu \mathrm{m}$ bump, which is caused by the $\mathrm{H}^{-}$opacity minimum in the atmospheres of cool stars. This feature is seen in the spectral energy distributions (SEDs) of all galaxies except active galactic nucleus (AGN) dominated ones and has long been considered a photometric redshift indicator (Wright et al. 1994; Simpson \& Eisenhardt 1999; Sawicki 2002). At $z>0.6$, the bump shifts beyond the $K$ band, and $f_{\nu}(3.6 \mu \mathrm{m})>f_{\nu}(2.2 \mu \mathrm{m})$. For $z>1.3$, the bump passes $3.6 \mu \mathrm{m}$, and $f_{\nu}(4.5 \mu \mathrm{m})>f_{\nu}(3.6 \mu \mathrm{m})$. Local, dusty starburst galaxies such as M82 also have red colors with $f_{\nu}(4.5 \mu \mathrm{m})>f_{\nu}(3.6 \mu \mathrm{m})$, and we need to require $f_{\nu}(3.6 \mu \mathrm{m})>$ $f_{\nu}(2.2 \mu \mathrm{m})$ to identify them.

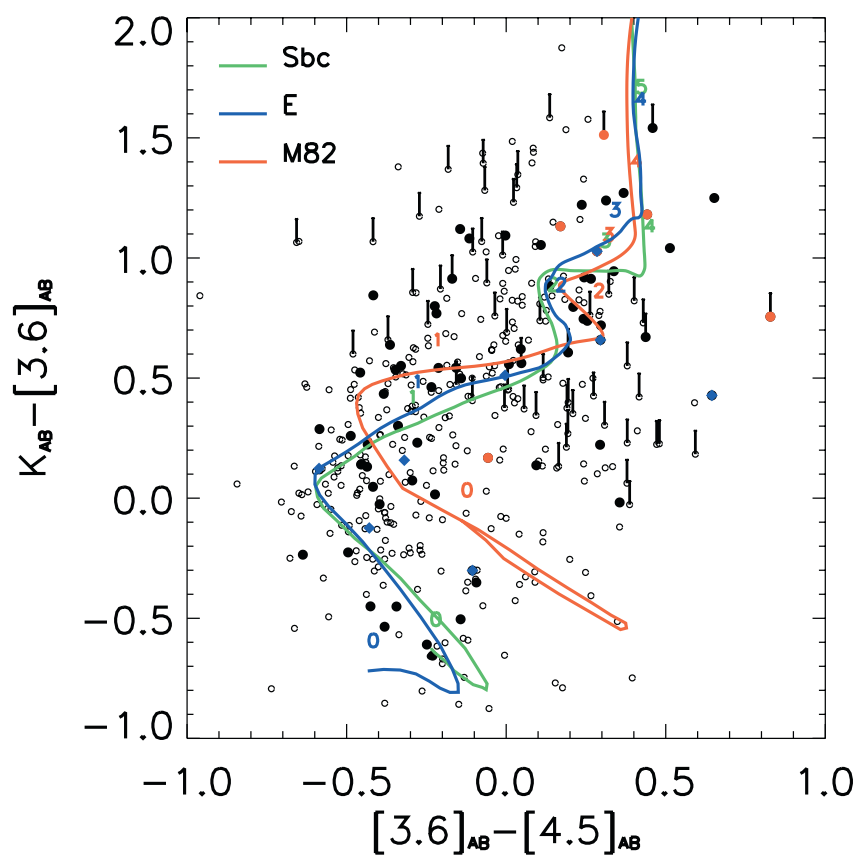

FIG. 1.-Color-color diagram for objects detected at 3.6 and $4.5 \mu \mathrm{m}$. Filled circles are objects also detected at $24 \mu \mathrm{m}$, open circles are remaining sample. Arrows indicate objects not detected at $K$, points are plotted at $3 \sigma$ limits. Blue diamonds denote XMM-Newton sources, and red circles denote SCUBA sources. Solid lines show color tracks for elliptical galaxies (blue), Sbc galaxies (green), and M82 (red). Expected colors for ellipticals and spirals are based on rest frame SEDs from Coleman et al. (1980) in visible light and $\mathrm{Lu}$ et al. (2003) in the infrared. M82 tracks are derived from the model of Granato et al. (2000) and ISO observations (Sturm et al. 2000). The tracks are derived by shifting the SED without adding any evolution; numbers marked along each track indicate redshift. In this diagram, galaxies in the upper left quadrant are likely to be at $0.6<z<1.3$, those in the upper right are likely to be at $z>1.3$, and galaxies in the two lower quadrants are likely to have $z<0.6$ with starburst galaxies to the right.

Figures 1 and 2 show the IRAC data along with the tracks followed by typical galaxy SEDs as redshift increases, and Table 2 summarizes the observed galaxy number distribution as a function of color, which imply redshift. Figure 1 shows that the observed colors are consistent with the model tracks. The most populated redshift bin in Table 2 for all four IRAC bands is $0.6<z<1.3$. This bin includes about $50 \%$ of all detected IRAC galaxies. Figure 1 shows a few sources with very red $K-$ [3.6] color. Wilson et al. (2004) found that these sources are actually the same extreme red objects as those detected in optical-near-infrared surveys using the $(R-K)>5$ color selection.

The color-color diagrams will not give accurate redshifts for AGNs, which do not exhibit the $1.6 \mu \mathrm{m}$ bump in their SEDs. X-ray observation is a good way of detecting AGNs. This field has nine XMM-Newton sources (Hasinger et al. 2001), whose colors are plotted along with other galaxies in Figures 1 and 2. These nine sources probably represent most of the AGNs in the field and constitute only a few percent of the galaxies detected. Thus, they should not affect our statistics on redshift distribution.

Unlike the XMM-Newton sources, whose color distribution fills the entire color space in Figures 1 and 2, most of the SCUBA source counterparts have colors indicating $z>1.3$. Using the deep radio counterpart positions to identify the SCUBA sources in the optical and Spitzer images, Egami et al. (2004) did a detailed analysis of SED for the SCUBA 


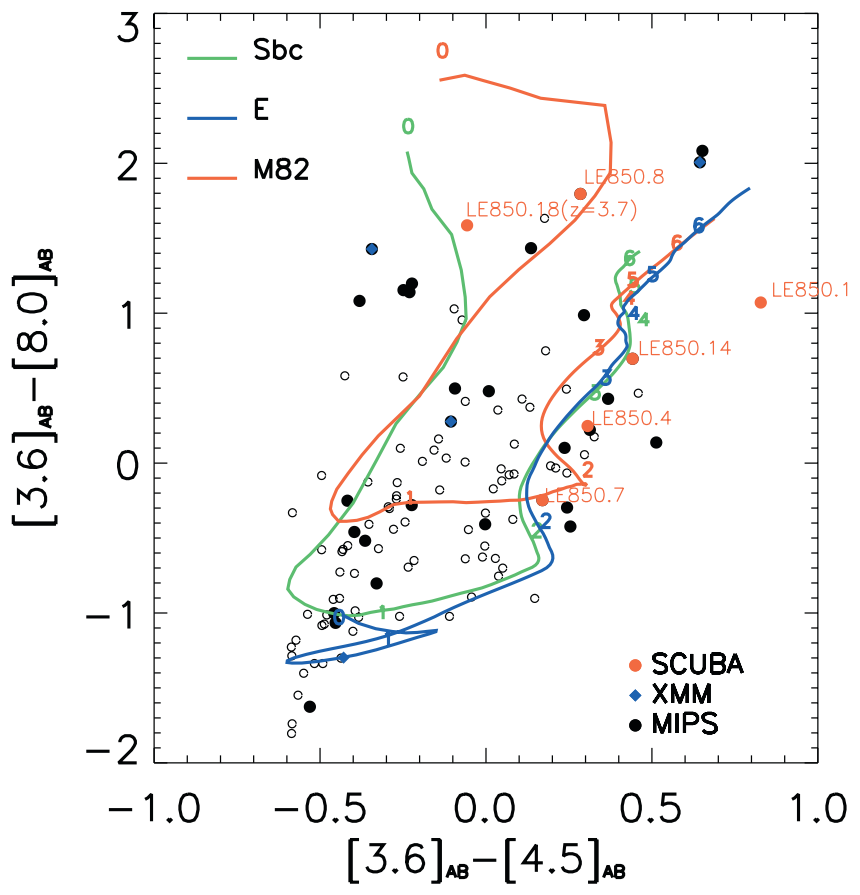

FIG. 2.-Color-color diagram for galaxies detected at $8 \mu \mathrm{m}$. Blue diamonds show XMM-Newton sources, red dots SCUBA show sources with numbers according to Scott et al. (2002), and black dots are objects detected at $24 \mu \mathrm{m}$. Open circles show the remaining sample galaxies. Color tracks are the same as those in Fig. 1.

sources. They found that LE $850.1,{ }^{9}$ LE 850.4 , LE 850.7 , and LE 850.14 have the $1.6 \mu \mathrm{m}$ bump in their SEDs. This implies that their SEDs are dominated by stellar light, and thus their IRAC colors following the galaxy color tracks well in Figures 1 and 2 with expected redshifts $z>2$. This is consistent with most of the SCUBA sources being dusty galaxies at $z>2$ (Chapman et al. 2003; Egami et al. 2004). LE 850.8 is the only object for which we have both SCUBA and XMM-Newton detection. Egami et al. (2004) found that LE 850.8 has a powerlaw continuum. Its IRAC colors are not close to any of the tracks in Figures 1 and 2. Another SCUBA source, LE 850.18, also has colors not close to any of the tracks and a power-law SED (Egami et al. 2004). In the deep $R$-band image, however, there are more than two objects within $3^{\prime \prime}$ radius centered on the radio counterpart positions for both LE 850.8 and LE 850.18. IRAC will not be able to resolve them if all components emit in the IRAC bands. Hence, we suspect foreground contamination in the IRAC flux densities in both cases and are cautious in using IRAC colors to classify both objects.

There are 32 sources in this field detected in the MIPS $24 \mu \mathrm{m}$ band at or above the $5 \sigma$ confidence level. Their IRAC counterparts show that half of the MIPS sources are at $z>1.3$.

${ }^{9}$ The full names according to SIMBAD are of the form [SFD2002] LHE N.
TABLE 3

Integrated Galaxy Light $\nu I_{\nu}\left(\mathrm{nW} \mathrm{m}{ }^{-2} \mathrm{sr}^{-1}\right)$ IN the IRAC Bands

\begin{tabular}{ccccc}
\hline \hline Objects & $3.6 \mu \mathrm{m}$ & $4.5 \mu \mathrm{m}$ & $5.8 \mu \mathrm{m}$ & $8.0 \mu \mathrm{m}$ \\
\hline All galaxies detected................... & 3.47 & 2.43 & 1.31 & 1.03 \\
SCUBA counterparts .............. & 0.05 & 0.05 & 0.05 & 0.04 \\
XMM-Newton counterparts ........ & 0.13 & 0.12 & 0.13 & 0.14 \\
MIPS counterparts .................. & 0.68 & 0.52 & 0.43 & 0.62 \\
\hline
\end{tabular}

Given the $5 \sigma$ limiting flux of $200 \mu \mathrm{Jy}$, these MIPS sources at $z>1.3$ have a minimum infrared luminosity of $10^{12} L_{\odot}$ and thus are LIRGs and ULIRGs (Le Floc'h et al. 2004).

The extragalactic background light (EBL) from both resolved and unresolved extragalactic sources is an indicator of the total luminosity of the universe (Madau \& Pozzetti 2000). Because the intermediate redshift galaxy population contributes most to the EBL (Elbaz et al. 2002), integral resolved galaxy light (IGL) in a deep survey should give a good lower limit on the EBL. Table 3 shows the results of integrating the fluxes, $\nu I_{\nu}$, from IRAC counterparts of objects identified by SCUBA (Scott et al. 2002), XMM-Newton (Hasinger et al. 2001), and MIPS $24 \mu \mathrm{m}$ images (Egami et al. 2004; Le Floc'h et al. 2004). The IGL at the 5.8 and $8.0 \mu \mathrm{m}$ are 1.31 and $1.02 \mathrm{nW} \mathrm{m}^{-2} \mathrm{sr}^{-1}$, very close to the ISO LW2 $(6.75 \mu \mathrm{m}) \mathrm{IGL}$, $1.7 \pm 0.5 \mathrm{nW} \mathrm{m}^{-2} \mathrm{sr}^{-1}$ (Altieri et al. 1999; Elbaz et al. 2002). Given that the XMM-Newton sources in this field are neither stars or clusters, it is safe to assume that most of them are AGNs (Lehmann et al. 2000; Alonso-Herrero et al. 2004). The light contributed by the XMM-Newton sources is constant in all four IRAC bands because of the flat SED of AGNs in the mid-infrared. Because the total light decreases with wavelength, the $X M M$-Newton source contribution increases from $3.7 \%$ at $3.6 \mu \mathrm{m}$ to $13.6 \%$ at $8 \mu \mathrm{m}$. This is consistent with the $15 \% \pm 5 \% X M M$-Newton source contribution found by the ISO survey (Fadda et al. 2002). As shown in Figure 2, most of the MIPS $24 \mu \mathrm{m}$ sources are at intermediate and high redshifts. Most of them must be LIRGs or even ULIRGs (Egami et al. 2004). The starburst light percentage increases from $20 \%$ at $3.6 \mu \mathrm{m}$ to $62 \%$ at $8 \mu \mathrm{m}$. AGNs and starburst galaxies together contribute about two-thirds of the IGL at $8 \mu \mathrm{m}$. The bulk of the light at 3.6 and $4.5 \mu \mathrm{m}$ appears to come from evolved stars in galaxies, while the 8 and $24 \mu \mathrm{m}$ light traces the star forming regions in galaxies. This explains the large contribution of the $24 \mu \mathrm{m}$ selected sample to the $8 \mu \mathrm{m}$ IGL.

\section{CONCLUSIONS}

Even with only 30 minutes observing time, IRAC detected 419 galaxies in a 4.7 $\times 4 .^{\prime} 7$ area in the Lockman Hole. Based on their colors, most of the detected galaxies are likely to be at intermediate redshifts $0.6<z<1.3$, but $\gtrsim 25 \%$ appear to be at $z>1.3 .30 \%$ of the $5.8 \mu \mathrm{m}$ sources and $50 \%$ of the $8.0 \mu \mathrm{m}$ sources show colors indicating higher redshifts $(z>1.3)$. The counterparts of the SCUBA sources have colors consistent

TABLE 2

Galaxies in Each IRAC Color Bin

\begin{tabular}{|c|c|c|c|c|c|c|c|}
\hline$K_{\mathrm{AB}}-[3.6]_{\mathrm{AB}}$ & {$[3.6]_{\mathrm{AB}}-[4.5]_{\mathrm{AB}}$} & Estimated Redshift & $3.6 \mu \mathrm{m}$ & $4.5 \mu \mathrm{m}$ & $5.8 \mu \mathrm{m}$ & $8.0 \mu \mathrm{m}$ & $24 \mu \mathrm{m}$ \\
\hline$>0 \ldots \ldots \ldots \ldots \ldots$ & $>0$ & $z>1.3$ & 113 & 122 & 35 & 24 & 16 \\
\hline$>0 \ldots \ldots \ldots \ldots \ldots$ & $<0$ & $0.6<z<1.3$ & 191 & 176 & 57 & 34 & 8 \\
\hline$<0 \ldots \ldots \ldots \ldots \ldots$ & $<0$ & $z<0.6$ & 101 & 81 & 27 & 22 & 8 \\
\hline$<0$ & $>0$ & $z<0.6$ & 14 & 24 & 1 & 0 & 0 \\
\hline
\end{tabular}


with galaxies at $z>1.3$. The integrated galaxy light seen in our sample is consistent with $1.7 \pm 0.5 \mathrm{nW} \mathrm{m} \mathrm{sr}^{-1}$ derived using the $I S O$ survey data at the $6.75 \mu \mathrm{m}$. The AGN fraction, as revealed by $X M M$-Newton, increases from $3.7 \%$ in the $3.6 \mu \mathrm{m}$ band to $13.6 \%$ in the $8.0 \mu \mathrm{m}$ band. The MIPS population, most of which are starburst galaxies at intermediate redshifts, contributes up to $60 \%$ in the $8.0 \mu \mathrm{m}$ band.
This work is based on observations made with the Spitzer Space Telescope, which is operated by the Jet Propulsion Laboratory, California Institute of Technology under NASA contract 1407. Support for this work was provided by NASA through contract 1256790 issued by JPL.

The Spitzer data (AOR 0006620160) from this paper are available through the electronic edition.
Alonso-Herrero, A., et al. 2004, ApJS, 154, 155

Altieri, B., et al. 1999, A\&A, 343, L65

Aussel, H., Elbaz, D., \& Cesarsky, C. J. 1999, Ap\&SS, 266, 307

Barmby, P., et al. 2004, ApJS, 154, 97

Chapman, S. C., Blain, A. W., Ivison, R. J., \& Smail, I. R. 2003, Nature, 422,369

Cimatti, A., et al. 2002, A\&A, 392, 395

Cohen, J. G., Hogg, D. W., Pahre, M. A., Blandford, R., Shopbell, P. L., \& Richberg, K. 1999, ApJS, 120, 171

Coleman, G. D., Wu, C.-C., \& Weedman, D. W. 1980, ApJS, 43, 393

Cowie, L. L., Songaila, A., Hu, E. M., \& Cohen, J. G. 1996, AJ, 112, 839

Dole, H., et al. 2001, A\&A, 372, 362

Egami, E., et al. 2004, ApJS, 154, 130

Elbaz, D., Cesarsky, C. J., Chanial, P., Aussel, H., Franceschini, A., Fadda, D., \& Chary, R. R. 2002, A\&A, 384, 848

Elbaz, D., et al. 1999, A\&A, 351, 37

Ellis, R. S. 1997, ARA\&A, 35, 389

Fadda, D., Flores, H., Hasinger, G., Franceschini, A., Altieri, B., Cesarsky, C. J., Elbaz, D., \& Ferrando, Ph. 2002, A\&A, 383, 838

Fazio, G. G., et al. 2004, ApJS, 154, 10

Genzel, R., \& Cesarsky, C. J. 2000, ARA\&A, 38, 761

Glazebrook, K., Peacock, J. A., Collins, C. A., \& Miller, L. 1994, MNRAS, 266,65

Granato, G. L., Lacey, C. G., Silva, L., Bressan, A., Baugh, C. M., Cole, S., \& Frenk, C. S. 2000, ApJ, 542, 710
REFERENCES

Hasinger, G., et al. 2001, A\&A, 365, 45

Huang, J.-S., Cowie, L. L., Gardner, J. P., Hu, E. M., Songaila, A.,\& Wainscoat, R. J. 1997, ApJ, 476, 12

Ivison, R. J., et al. 2002, MNRAS, 337, 1 2004, ApJS, 154, 124

Kessler, M. F. 1996, BAAS, 188, 5201

Koo, D. C., \& Kron, R. G. 1992, ARA\&A, 30, 613

Lehmann, I., et al. 2000, A\&A, 345, L35

Le Floc'h, E., et al. 2004, ApJS, 154, 170

Lu, N., et al. 2003, ApJ, 58, 199

Madau, P., \& Pozzetti, L. 2000, MNRAS, 312, L9

Oliver, S. J., et al. 1997, MNRAS, 289, 471

Sato, Y., et al. 2003, A\&A, 405, 833

Scott, S. E., et al. 2002, MNRAS, 331, 817S

Sawicki, M. 2002, AJ, 124, 3050

Simpson, C., \& Eisenhardt, P. 1999, PASP, 111, 691

Smail, I., Ivison, R. J., Blain, A. W., \& Kneib, J.-P. 2002, MNRAS, 331, 495

Steidel, C. C., Adelberger, K. L., Shapley, A. E., Pettini, M., Dickinson, M., \& Giavalisco, M. 2003, ApJ, 592, 728

Sturm, E., Lutz, D., Tran, D., Feuchtgruber, H., Genzel, R., Kunze, D., Moorwood, A. F. M., \& Thornley, M. D. 2000, A\&A, 358, 481

Thompson, D., et al. 1999, ApJ, 523, 100

Wilson, G., Kaiser, N., Luppino, G. A., \& Cowie, L. L. 2001, ApJ, 555, 572

Wilson, G., et al. 2004, ApJS, 154, 107

Wright, E. L., Eisenhardt, P., \& Fazio, G. 1994, BAAS, 184, 2503 